Logic and Logical Philosophy

Volume 17 (2008), 5-7

DOI: $10.12775 /$ LLP.2008.001

\title{
FROM THE GUEST EDITORS
}

On the $28^{\text {th }}$ of October, 2006, Alexander Vladimirovich Kuznetsov, so is his full name, would have turned 80. Although belated, the editorial board of Logic and Logical Philosophy, we, the editors and contributors of the present issue, and other members of the logic community mark this event with the present issue. Most of those who contributed to it knew Kuznetsov in person and/or were influenced by him or by his ideas, which very often resided in somebody else's papers or became part of a scientific folklore. But, to be sure, there are even more mathematicians and logicians who have been witnesses to his scientific activity and/or have taken advantage of its results.

The issue is organized in two distinct segments. First we present three articles, A. Y. Muravitsky, "Alexander Vladimirovich Kuznetsov", A. I. Citkin, "A mind of a non-countable set of ideas", and A. Y. Muravitsky, "The contribution of A. V. Kuznetsov to the theory of modal systems and structures", which are mostly of a historical character. The first article is a brief outline of Kunzetzov's growth as a "mathematical logician", as he regarded himself and as states the short inscription on his grave on the city cemetery in Chişinău, Moldova, within the development of the Soviet mathematical logic of his lifetime. Kuznetsov's understanding of this term, as well as some of his other ideas, are explained in the second article. Also presented there is Kuznetsov's nonstandard manner in handling mathematical problems, including both successful and unsuccessful attempts. The third article discusses Kuznetsov's activity and contributions near the end of his active scientific life.

The second segment comprises the following original scientific contributions:

Sergei Artemov and Elena Nogina, in the article "Topological semantics of justification logic", extend a well-known Tarski's topological interpretation 
of $\mathbf{S} \mathbf{4}$ to a justification logic obtained from modal logic by adding a new type of formulas $t: F$ which reads as $t$ is a justification for $F$.

The article "The Kuznetsov-Gerčiu and Rieger-Nishimura logics. The boundaries of the finite model property" by Guram Bezhanishvili, Nick Bezhanishvili and Dick De Jongh contains a detailed study of finite model property FMP in the extensions of Kuznetsov-Gerčiu logic KG. The authors suggest a new technique based on the relational semantics, which allows one to prove that there is a continuum of $\mathbf{K G}$ extensions without FMP. The paper contains a simplified proof of two results by Gerčiu on that all extensions of Rieger-Nishimura logic have FMP and on characterizing the only extension of $\mathbf{K G}$ that bounds FMP for extensions of $\mathbf{K G}$. The variations of $F M P$ and locally tabular $\mathbf{K G}$-extensions are treated also.

Leo Esakia and Revaz Grigolia describe the free cyclic algebra over the variety of Solovay algebras in the paper "Formulas of one propositional variable in intuitionistic logic with the Solovay modality".

The article "Interpolation and implicit definability in extensions of the provability logic" by Larisa Maksimova is devoted to the investigation of various variants of the interpolation property and the explicit definability property. The author gives an extensive survey of interrelationships among various versions of both properties regarding some extensions of the provability logic GL. She focuses especially on the restricted interpolation property and the deductive interpolation property, showing that the former implies the latter in modal logics of some designated class.

Vladimir Rybakov, in the paper "Discrete linear temporal logic with current time point clusters, deciding algorithms", proves the decidability of the logic of discrete linear time with current time point clusters and considers the admissibility problem for this logic.

The paper "A modal approach to dynamic ontology: modal mereotopology", by Dimiter Vakarelov, considers a mereology system with additional relations of topological nature like contact relation. The author shows that modal operators can be used to define some new mereological and mereotopological relations with dynamic nature like stable part-of and stable contact. It is proved that the resulting system has the finite model property.

Heinrich Wansing and Karoline Semmeling, in "From BDI and stit to bdi-stit logic", develop a system which combines the approach by Rao and Georgeff to logics of belief, desires, and intensions with one of the most prominent multi-agent logics, so called stit-logic. 
The editors would like to take the opportunity to thank everyone who in one way or another positively reacted to mark a birthday anniversary of one of the eminent mathematical logicians of the second half of the 20th century. Also, we are especially grateful to E. A. Pavlova, E. A. Kuznetsov, S. I. Adian, A. A. Muchnik and B. A. Trakhtenbrot for sharing their memories of Alexander V. Kuznetsov.

A. Muravitsky

S. Odintsov 